\title{
A New Look at Poor Groups of Galaxies
}

\author{
Ann I. Zabludoff \\ UCO/Lick Observatory and Astronomy Department, University of \\ California, Santa Cruz, CA 95064
}

\begin{abstract}
We use a new fiber spectroscopic survey of 12 nearby, poor groups of galaxies to examine the dynamics and evolution of galaxies in these common, but poorly studied, environments. Some of our conclusions are: (1) The nine groups in our sample with diffuse X-ray emission are in fact bound systems with at least 20-50 group members with absolute magnitudes as faint as $M_{B} \sim-14+5 \log _{10} h$. (2) Galaxies in each X-ray-detected group have not all merged together because a significant fraction of the group mass lies outside of the galaxies and in a common halo, thereby reducing the rate of galaxy-galaxy interactions. (3) The similarity of the recent star formation histories and the fraction of early type galaxies in some poor groups to those in rich clusters suggest that cluster-specific environmental effects may not play a dominant role in the recent evolution of cluster galaxies. The evolution of group and cluster members may be driven instead by galaxy-galaxy interactions, which are likely to occur with equal frequency in field groups and in groups that have recently fallen into clusters (i.e., subclusters).
\end{abstract}

\section{Introduction}

Most galaxies in the nearby universe, including our own Galaxy, belong to poor groups of galaxies. Despite the ubiquity of the group environment, we know little about the internal dynamics of groups and the evolution of group galaxies outside of the Local Group. To learn whether the diverse and detailed results on the Local Group presented at this meeting apply elsewhere, we must also survey other poor groups.

Because poor groups are apparent systems of fewer than five bright $\left(\leq M^{*}\right)$ galaxies, past studies have been hampered by small number statistics. Some of the critical, unanswered questions are (1) whether most poor groups are real systems instead of chance projections of galaxies along the line-of-sight, (2) why many poor groups, with their favorable environments for galaxy-galaxy mergers, have survived until now, (3) and how galaxies evolve in groups, where the collisional effects of the intragroup gas and the tidal influences of the global potential are weaker than in rich clusters. The advent of multi-object spectroscopy now makes it possible to address such questions in unprecedented detail. In this talk, I discuss the first results obtained in collaboration with John Mulchaey (Carnegie Observatories) from a fiber spectroscopic survey of 12 nearby, poor 
groups (Zabludoff \& Mulchaey 1998a; Mulchaey \& Zabludoff 1998; Zabludoff \& Mulchaey 1998b).

\section{Poor Groups: What are They?}

\subsection{Three Classes of Groups}

Poor groups in the literature have been identified optically and fall into several classes that can be distinguished by their X-ray properties and bright galaxy morphologies. Groups with detectable, hot intra-group gas typically have a giant $\left(\leq M^{*}-1\right)$ elliptical that is the brightest group galaxy (BGG) and that lies near or on the peak of the smooth, symmetric X-ray emission (Mulchaey et al. 1996). In contrast, groups without extended X-ray emission tend to optically resemble the Local Group, which consists of a few bright, late-type galaxies and their satellites. If some non-X-ray-detected, late-type-dominated groups evolve into groups with a central, giant elliptical and a detectable intra-group medium, then groups in transition may form a third class of objects. We would expect the cores of such systems to have signatures of recent dynamical evolution, including interacting galaxies and X-ray gas that does not coincide with the galaxies. To construct our sample, we select poor groups from these three classes that also have complementary X-ray images.

The sample consists of 12 nearby $\left(1500<c z<8000 \mathrm{~km} \mathrm{~s}^{-1}\right)$, opticallyselected groups from the literature for which there are existing, sometimes serendipitous, pointed ROSAT/PSPC X-ray observations: NGC 533, NGC 5129, NGC 5946, NGC 4325, NGC 741, NGC 2563, HCG 42, HCG 62, HCG 90, NGC 664 , NCG 491, and NGC 7582. Nine of the groups are X-ray-detected. HCG 90 (Hickson 1982), a possible transitional object with several interacting galaxies in its core (Longo et al. 1994), is only marginally X-ray-detected (Ponman et al. 1996). It is important to stress that our group sample is biased with respect to published group catalogs, in which less than half of the groups are $\mathrm{X}$-ray-detected. The sample is weighted toward X-ray groups, because of the likelihood that they are bound systems and the most evolved poor groups.

\subsection{Bound or Superpositions?}

The issue of whether many poor groups, even those identified from redshift surveys, are bound systems instead of chance superpositions of galaxies along the line-of-sight has been a puzzle. The existence of one poor group, our Local Group, is unchallenged. In contrast, Ramella et al. (1989) show that $\sim 30 \%$ of groups of three or four galaxies in the CfA Redshift Survey (Huchra et al. 1995) are probably unbound, geometric projections. The detection of a significant population of fainter members would be an important first step in identifying poor groups that are real.

Using the Las Campanas fiber spectrograph designed by Steve Shectman, we measured the $\sim 100$ brightest galaxies projected within $1.5^{\circ} \times 1.5^{\circ}$ of the center of each of the 12 sample groups. The radial velocity distributions for galaxies in the fields of the nine X-ray-detected groups each reveal $\sim 20-50$ group members down to absolute magnitudes of $M_{B} \sim-14$ to $-16+5 \log _{10} h$. The surprisingly large membership, the central concentration of early type galaxies, the similarity 
of the BGG's position and orientation to those of the diffuse X-ray emission, the consistency of the optical velocity dispersion and the X-ray temperature, and the short crossing times ( $\leq 0.05$ of a Hubble time) of the X-ray groups suggest that they are bound systems, not geometric superpositions of galaxies, and that the group cores are close to virialization or virialized.

Because we do not detect diffuse X-ray emission and find only 5-8 members in the three non-X-ray-detected groups, we are unable to determine their dynamical state. The non-X-ray groups, which consist of one or two $M^{*}$ or brighter spirals with several fainter galaxies that may be satellites, are morphologically akin to the Local Group (although our samples are not sufficiently deep to ascertain whether any group has a dwarf spheroidal population like that of the Local Group; van den Bergh 1992). If the non-X-ray groups are dynamically similar to the Local Group, they are bound (see Zaritsky 1994). Our current data do not exclude this possibility - the velocity dispersions of the non-X-ray groups are consistent with the upper limits on their X-ray luminosities (Mulchaey \& Zabludoff 1998).

\section{Poor Groups: Why are They?}

If some poor groups are bound systems, then another critical question is why they exist at all. Poor groups have higher galaxy densities than the field and lower velocity dispersions than cluster cores, making them favorable sites for galaxy-galaxy mergers (Barnes 1985). The likelihood of mergers and the short group crossing times ( $\leq 0.05$ of a Hubble time) suggest that most groups should have already merged into one object. Therefore, either bound groups are collapsing for the first time like the Local Group (Zaritsky 1994) or only a small fraction of the group mass is tied to the galaxies, lowering the rate of galaxy-galaxy interactions relative to a galaxy-dominated system and allowing the group to survive many crossing times (Governato et al. 1991; Bode et al. 1993; Athanassoula et al. 1997). To address this issue by measuring the underlying mass distribution of poor groups, we use the improved statistics of our spectroscopic survey.

The velocity dispersion of the combined group sample does not decrease significantly with radius from the central $\sim 0.1 h^{-1} \mathrm{Mpc}$ to at least $\sim 0.5 h^{-1}$ $\mathrm{Mpc}$, in contrast to the more than factor of two decrease that would be observed if the entire mass were concentrated within $0.1 h^{-1} \mathrm{Mpc}$. The extended mass is either in the galaxies, in a common halo through which the galaxies move, or in both the galaxies and a diffuse halo. If all the mass were tied to the galaxies, most of the mass would be associated with the bright, central elliptical in those groups in which the BGG dominates the light. For groups with a few galaxies that have luminosities comparable to the BGG, the velocity dispersion would be increased at large radii by subgroups consisting of a massive galaxy and the subgroup members that are orbiting it. If this picture were accurate, we would expect the velocity dispersion profiles of groups with several comparably bright galaxies to be shallower than those in which the BGG is dominant. However, the combined velocity dispersion profile of a subsample of two groups (HCG 42 and NGC 741) in which the BGG dominates the light (i.e., the BGG luminosity exceeds the combined luminosity of the other $M^{*}$ or brighter galaxies) is indistinguishable 
from that of the entire sample. Therefore, we conclude that most of the group mass lies in a smooth, extended dark halo.

This result argues that poor groups survive longer than predicted by models in which all the mass is tied to individual galaxies and may explain why so many poor groups are observed in lieu of single merger remnants.

\section{Poor Groups: How do Galaxies Evolve in Them?}

\subsection{Distribution of Early Type Fractions}

The factors that might affect the evolution of galaxies in poor groups are different from those present in rich clusters. If clusters evolve hierarchically by accreting poor groups of galaxies (subclusters), members of an infalling group have recently experienced the hot, dense cluster environment for the first time. Therefore, galaxies in poor groups in the field are a control sample for understanding the factors that influence the evolution of their counterparts in subclusters.

For example, we can compare the morphologies and recent star formation histories of galaxies in the subclusters of complex clusters like Coma (Caldwell \& Rose 1997) with those of galaxies in poor field groups. Differences between the samples would argue that cluster environment is important in transforming galaxies at the present epoch. On the other hand, the lack of such differences would suggest, as the simplest explanation, either that star formation and morphology are influenced by mechanisms present in both field groups and subclusters, such as galaxy-galaxy encounters, or that the effects of environment on galaxies are insignificant compared with conditions at the time of galaxy formation.

Despite the usefulness of group galaxies as a control sample, their properties, especially at faint magnitudes, are not well-known. Past work has included only the four or five brightest galaxies, which biases samples toward ellipticals, and has targeted only the central $\leq 0.3 h^{-1} \mathrm{Mpc}$, where early types concentrate. Therefore, to compare the morphologies and star formation histories of group and cluster members, we must sample both environments to similar physical radii and absolute magnitude limits.

In the X-ray groups, the early type fraction $(f)$ ranges widely from $\sim 55 \%$ (HCG 62, NGC 741, and NGC 533) to 25\% (e.g., NGC 2563). The latter value is characteristic of the field ( $\sim 30 \%$; Oemler 1992$)$. We find no early types among the 6-8 galaxies in each of the three non-X-ray groups. The early type fractions of $\sim 55 \%$ in NGC 533, NGC 741, and HCG 62 are most surprising, because they are consistent with those of rich clusters for similar physical radii and absolute magnitude limits ( 0.55-0.65; Whitmore et al. 1993).

\subsection{Early Type Fraction vs. Velocity Dispersion}

The correlation between early type fraction and group velocity dispersion is significant at the $>0.999$ level. The form of the relation cannot be the same for rich clusters - our fit to the group data predicts that the early type fraction in a $\sigma_{r} \sim 1000 \mathrm{~km} \mathrm{~s}^{-1}$ cluster is an unphysical $f=124 \%$ ! Therefore, the relation must turn up between the poor group and rich cluster regimes. The group $f-\sigma_{r}$ relation implies either that galaxy morphology is set by the local potential size 
at the time of galaxy formation (Hickson, Huchra, \& Kindl 1988) and/or that $\sigma_{r}$ and $f$ increase as a group evolves (Diaferio et al. 1995).

The early type fractions in our highest velocity dispersion groups $\left(\sigma_{r} \sim 400\right.$ $\mathrm{km} \mathrm{s}^{-1}$ ) are as high as in rich clusters. If some early type galaxies are evolved merger remnants, then the galaxy populations of higher velocity dispersion groups are more evolved on average. At some point in the group's evolution, perhaps at a velocity dispersion near $400 \mathrm{~km} \mathrm{~s}^{-1}$, any morphological evolution resulting from galaxy mergers ceases, and the fraction of merger remnants in poor groups and rich clusters is comparable. The implicit upturn in our $f-\sigma_{r}$ relation suggests such a saturation point.

Alternatively, the similarity of some group and cluster early type fractions, and the steepening of the $f-\sigma_{r}$ relationship at high $\sigma_{r}$, might arise from conditions at the time of galaxy formation. For example, it is possible that poor groups such as NGC 533 and rich clusters like Coma begin as similar mass density perturbations with correspondingly similar galaxy populations. In this simple picture, NGC 533 does not develop a cluster-size potential, because its field lacks the surrounding groups that Coma later accretes.

In summary, the cluster-like fraction of early type galaxies in NGC 533, NGC 741, and HCG 62 indicate that clusters are not the only environments with copious quantities of $\mathrm{E}$ and $\mathrm{S} 0$ galaxies. The simplest explanation is either that fluctuations in the initial conditions permitted early types to form in these groups' comparatively low velocity dispersion, low galaxy density environments or that the galaxies' subsequent evolution was the product of a mechanism, such as galaxy-galaxy interactions, common to both groups in the field and groups that become subclusters. Although additional environmental mechanisms may affect the evolution of cluster galaxies, such cluster-specific processes are not required to explain the current data. A cluster that evolves hierarchically from subclusters with the properties of NGC 533, NGC 741, and HCG 62 will have, at least initially, a similar galaxy population.

\subsection{Star Formation in Early Types}

The star formation histories of galaxies in poor groups provide additional insight into the environmental factors that may influence the evolution of galaxies. One approach is to examine the spectra of the early types for evidence of on-going star formation or of a young stellar population. We can then compare the fraction of E and S0 group members that have recently formed stars with a sample from rich clusters with complex structure.

Star formation is on-going or has ended within the last $\sim 2 \mathrm{Gyr}$ in eight of the 64 early type group members for which we have spectra (12\%). This fraction is roughly the same for clusters, such as Coma, with infalling groups ( 15\%; Caldwell \& Rose 1997). This result, and the similarity of the early type fractions of some poor groups to rich clusters, suggests that an environmental mechanism present in both groups and subclusters may be responsible. For example, galaxy-galaxy encounters can produce bursts of star formation (e.g., Londsdale et al. 1984; Kennicutt et al. 1987; Sanders et al. 1988) in which the gas is consumed or stripped away.

Although our observations of poor groups suggest that the effects of cluster environment are not necessary to produce the early type fractions and star for- 
mation episodes of nearby clusters, we suspect that the star formation histories of group and subcluster galaxies will begin to deviate after the subcluster and cluster mix. Proposed gas removal processes including ram pressure stripping and the tidal limitation of galaxy halos, which are more efficient in clusters than in groups, may eventually suppress star formation in cluster galaxies. Comparative studies of the HI content of field, group, and cluster galaxies will help to resolve this issue.

Acknowledgements. This research was supported by grants from the NSF and NASA.

\section{References}

Athanssoula, E., Makino, J., Bosma, A. 1997, MNRAS, 286, 825

Barnes, J. 1985, MNRAS, 215, 517

Bode, P.W., Cohn, H.N., Lugger, P.M. 1993, ApJ, 416, 17

Caldwell, N., Rose, J. 1997, AJ, 113, 492

Diaferio, A., Geller, M., Ramella, M. 1995, AJ, 109, 2293

Governato, F., Bhatia, R., Chincarini, G. 1991, ApJ, 371, L15

Hickson, P., Huchra, J., Kindl, E. 1988, ApJ, 331, 64

Hickson, P. 1982, ApJ, 255, 382

Huchra, J.P., Geller, M.J., Corwin, H. 1995, ApJS, 99, 391

Kennicutt, R.C., Roettiger, K.A., Keel, W.C., Van der Hulst, J.M., Hummel, E. 1987, ApJ, 93, 1011,

Lonsdale, C.J., Persson, S.E., Matthews, K. 1984, ApJ, 287, 95

Longo, G., Busarello, G., Lorrenz, H., Richter, G., Zaggia, S. 1994, A\&A, 282, 418

Mulchaey, J.S., Zabludoff, A.I. 1998, ApJ, 496, 73

Mulchaey, J., Davis, D., Mushotzky, R., Burstein, D. 1996, ApJ, 456, 80

Oemler, A. 1992, in: Clusters and Superclusters of Galaxies, (ed.) A.C. Fabian (Dordrecht: Kluwer), p. 29

Ponman, T., Bourner, P., Ebeling, H., Bohringer, H. 1996, MNRAS, 283, 690

Ramella, M., Geller, M.J., Huchra, J.P. 1989, ApJ, 344, 57

Sanders, D.B., Soifer, B.T., Elias, J.H., Matthews, K., Madore B.F. 1988, ApJ, 325,74

van den Bergh, S. 1992, A\&A, 264, 75

Whitmore, B., Gilmore, D., Jones, C. 1993, ApJ, 407, 489

Zabludoff, A.I., Mulchaey, J.S. 1998a, ApJ, 496, 39

Zabludoff, A.I., Mulchaey, J.S. 1998b, ApJ, 498, L5

Zaritsky, D. 1994, in: The Local Group: Comparative and Global Properties, (eds.) A. Layden, R.C. Smith, \& J. Storm, ESO Conference and Workshop Proceedings No. 51, p. 187 


\section{Discussion}

Terndrup: Could you please elaborate on your remarks about the coupling between the elliptical fraction in groups and group environment?

Zabludoff: We observe a strong correlation between group early-type fraction and velocity dispersion. This correlation could result either from an increase in the early type fraction and velocity dispersion as a group evolves, where galaxy morphologies change due to a mechanism such as galaxy-galaxy mergers, or from conditions at the time of galaxy formation that tie the E and S0 fraction to the potential well depth early on. One point suggesting that group galaxy evolution is affected by environment at later epochs is that this correlation has a more shallow slope than that for rich clusters. In other words, there seems to be a saturation point at which early type fraction stops increasing with velocity dispersion. This point is at about 400 to $500 \mathrm{~km} \mathrm{~s}^{-1}$, which is about the velocity dispersion that a poor group would require such that an $M^{*}$ galaxy would experience a merger within a Hubble time. Therefore, it is possible that mergers cause some evolution in the early type fraction of poor groups and cease to be effective in richer groups and clusters. 